\title{
Review
}

\section{S100A4 and Metastasis}

\section{A Small Actor Playing Many Roles}

\author{
Kjetil Boye ${ }^{\star \dagger}$ and Gunhild M. Mælandsmo* \\ From the Department of Tumor Biology, Institute for Cancer \\ Research* and Department of Oncology, ${ }^{\dagger}$ The Norwegian Radium \\ Hospital, Oslo University Hospital, Oslo, Norway
}

\begin{abstract}
The calcium-binding protein S100A4 promotes metastasis in several experimental animal models, and S100A4 protein expression is associated with patient outcome in a number of tumor types. $\$ 100 \mathrm{~A} 4$ is localized in the nucleus, cytoplasm, and extracellular space and possesses a wide range of biological functions, such as regulation of angiogenesis, cell survival, motility, and invasion. In this review, we summarize the evidence connecting S100A4 and cancer metastasis and discuss the mechanisms by which S100A4 promotes tumor progression. (Am J Pathol 2010, 176:528-535; DOI: 10.2353/ajpath.2010.090526)
\end{abstract}

S100A4, also known as mts1, p9Ka, FSP1, CAPL, calvasculin, pEL98, metastasin, 18A2, and 42A, was cloned in the 1980 s and early 1990 s from various cell systems. ${ }^{1,2}$ It belongs to the $\mathrm{S} 100$ protein family, which consists of at least 21 different members. ${ }^{3}$ S100 proteins are $\mathrm{Ca}^{2+}$. binding, low molecular mass proteins (10 to $12 \mathrm{kDa}$ ) that generally exist as homo- or heterodimers within cells. ${ }^{4}$ Multimeric forms have also been reported for certain family members, ${ }^{4}$ and these functional states appear to be associated with extracellular activity. S100 proteins possess no enzymatic activity; instead, they exert their effects by interacting with and modulating the activity of other proteins. ${ }^{2,4}$ Altered expression levels of S100 proteins have been described in several human diseases, such as cancer, inflammatory disorders, cardiomyopathies, and neurodegenerative conditions, ${ }^{5}$ and S100A4, in particular, has gained increasing attention over the last two decades because of its metastasis-promoting properties. In this review, the evidence implicating S100A4 in the process of cancer metastasis is summarized. Apart from the following introduction, other aspects of S100A4 biology, such as biochemical characterization, transcriptional regulation, and studies on nonmalignant diseases, are only addressed if relevant to the understanding of cancer metastasis.

\section{Gene Structure and Transcriptional Regulation}

The human S100A4 gene is located in a frequently rearranged gene cluster on chromosome 1q21 and is composed of four exons, of which the first two are noncoding. ${ }^{1}$ The 101-amino acid protein has a molecular mass of approximately $11.5 \mathrm{kDa}$ and is characterized by the presence of two $\mathrm{Ca}^{2+}$-binding EF-hands. Upon $\mathrm{Ca}^{2+}$ binding, S100A4 undergoes a conformational change, forming a hydrophobic pocket essential for the recognition of target proteins. $^{6}$

The transcriptional regulation of S100A4 has been most extensively studied using murine cell lines. In mouse adenocarcinoma cells, an enhancer region located within the first intron controls S100A4 transcription, ${ }^{7}$ whereas in other cell systems upstream regulatory elements have also been identified. ${ }^{1}$ In human cells, methylation status, $\beta$-catenin, and extracellular factors have been reported to affect the expression of S100A4. ${ }^{1,2,8}$

\section{Subcellular Localization and Tissue Distribution}

S100A4 is localized in the nucleus, cytoplasm, and the extracellular space. Cytoplasmic expression is evident in the vast majority of S100A4-expressing cells, and intracellular concentrations may be as high as $10 \mu \mathrm{mol} / \mathrm{L}$ in certain cells. ${ }^{6}$ Nuclear localization was first demonstrated by Flatmark et $\mathrm{al}^{9}$ in a panel of primary tumor samples from patients with colorectal cancer using immunohistochemistry and subcellular fractionation experiments, and this finding has later been corroborated by a number of

\footnotetext{
Supported by the Norwegian Cancer Society (C99026 to K.B. and G.M.M.) and the National Program for Research in Functional Genomics of the Research Council of Norway (158954/S10 to G.M.M.).

Accepted for publication September 29, 2009

Address reprint requests to Kjetil Boye, M.D., Ph.D., Department of Tumor Biology, Institute for Cancer Research, The Norwegian Radium Hospital, Oslo University Hospital, N-0310 Oslo, Norway. E-mail: kjetil. boye@rr-research.no.
} 
reports demonstrating expression of S100A4 in the nuclei of both normal and neoplastic cells. ${ }^{10-13}$ Growing evidence indicates that $\mathbf{S 1 0 0}$ family members are secreted to the extracellular space, but the mechanism of secretion is still obscure. ${ }^{14}$ In recent years, release of S100A4 from tumor cells, macrophages, and fibroblasts in culture has been demonstrated. ${ }^{12,15-18}$ Secretion of S100A4 in vivo is supported by its presence in tumor interstitial fluid (ie, patient tumor samples incubated in saline in vitro) and increasing S100A4 levels in the serum of aging S100A4 transgenic mice. ${ }^{15,16}$

To our knowledge, no comprehensive investigation of S100A4 protein expression in normal human tissue has been undertaken. Nevertheless, several investigations have reported the expression of S100A4 in various normal human cell types, including fibroblasts, ${ }^{18,19}$ monocytes ${ }^{16}$ macrophages, ${ }^{20}$ T lymphocytes, ${ }^{16}$ neutrophilic granulocytes, ${ }^{16}$ and endothelial cells. ${ }^{10}$ Furthermore, several immunohistochemical analyses have demonstrated the expression of S100A4 in lymphocytes, macrophages, endothelium, and smooth muscle cells of vessel walls adjacent to tumor tissue in various organs. ${ }^{9,21-23}$

During the last decade, S100A4 has been widely accepted as a fibroblast-specific marker and hence used in a number of different investigations in humans and rodents. However, as summarized above, the existing literature contains several reports demonstrating expression of S100A4 in a wide range of normal cell types. Investigations of S100A4 protein expression should be carefully considered owing to possible nonspecific binding of antiS100A4 antibodies. Because S100 proteins are relatively homologous, it is reasonable to assume that antibodies directed against specific family members show some cross-reactivity. However, the data presented have been confirmed using several different antibodies, both monoclonal and polyclonal, and for certain cell types, including monocytes, macrophages, and T lymphocytes, protein expression data are substantiated by abundant expression of S100A4 mRNA. ${ }^{20,24}$ The assumption that S100A4 expression is restricted to fibroblasts is clearly misleading, and the conclusions drawn on this basis should be questioned.

\section{Binding Partners}

S100A4 has no enzymatic activity and exerts its functions mainly through interaction with other proteins. Intracellularly, S100A4 exists predominantly as a symmetric homodimer, facilitating the simultaneous binding and functional cross-linking of two homologous or heterologous target proteins. ${ }^{25}$ Similar to other S100 proteins, a number of binding partners for S100A4 have been identified, including actin, nonmuscle myosin IIA and IIB, tropomyosin, p53, liprin $\beta 1$, methionine aminopeptidase $2, \mathrm{CCN} 3$, S100A1, p37, and septin 2, 6, and 7 (Figure 1A). ${ }^{25,26}$ Spatial and temporal colocalization of S100A4 and its proposed target proteins is confirmed in only a few cases, and the functional correlates of S100A4-target protein interactions are described infrequently. Consequently, not all interactions demonstrated in vitro are necessarily biologically relevant. Because the molecular in- teractions between S100A4 and its intracellular target proteins have been thoroughly reviewed recently, ${ }^{25,26}$ a complete discussion on this subject will not be presented here.

In the extracellular space, S100A4 has been reported to interact with the cell surface receptors receptor for advanced glycation end products (RAGE) and annexin II, as well as heparan sulfate proteoglycans (Figure 1B). ${ }^{10,27,28}$ In general, the biological activity of extracellular S100A4 is associated with multimeric forms of the protein, ${ }^{15,27,29}$ although extracellular activity has been reported also for dimeric S100A4. ${ }^{28}$

\section{S100A4 and Metastasis: Experimental Evidence}

Metastasis is generally defined as the spread of malignant cells from the primary tumor through the circulation to establish secondary growth in a distant organ. Some of the experimental animal models used to causally implicate S100A4 in the metastatic process are based on intravenous or intracardial injection of cells with manipulated levels of S100A4. Such models only encompass the postinvasion steps in the metastatic cascade, whereas other assays that have been used depict the entire metastatic process through injection of tumor cells to form primary tumors, either into orthotopic or nonorthotopic sites (eg, subcutaneous injection). For simplicity, all models are herein referred to as experimental metastasis assays, but the reader should keep in mind the limitations discussed above.

Since the cloning of S100A4 from highly metastatic murine mammary carcinoma cells, ${ }^{30}$ its importance in the process of cancer metastasis has been corroborated by several experimental approaches, and in the following text, the experimental evidence implicating S100A4 in the progression of malignant cells to a metastatic phenotype is summarized.

Overexpression of S100A4 in a benign rat mammary epithelial cell line was shown to promote subcutaneous tumor growth and metastasis to the lungs and lymph nodes, ${ }^{31}$ and, correspondingly, the nonmetastatic human breast cancer cell line MCF-7 acquired a metastatic phenotype upon S100A4 transfection. ${ }^{32}$ Furthermore, decreased expression of S100A4 in highly metastatic human osteosarcoma cells produced a significant suppression of experimental metastasis formation after intracardial injection in rats, ${ }^{33}$ and S100A4 antisense-transfected Lewis lung carcinoma cells displayed reduced metastatic capacity upon tail vein injection in syngeneic mice. ${ }^{34}$ In an orthotopic model of bladder cancer, S100A4 overexpression induced lymph node and lung metastasis, ${ }^{35}$ and increased metastatic growth was also observed after intrasplenal and intracardial injection of colon cancer cells with high levels of S100A4 induced by transfection of mutant $\beta$-catenin. ${ }^{8}$

Further evidence emphasizing the central role of S100A4 in tumor progression and metastasis comes from genetically engineered mice. In two transgenic mouse models, animals overexpressing S100A4 were phenotyp- 
A

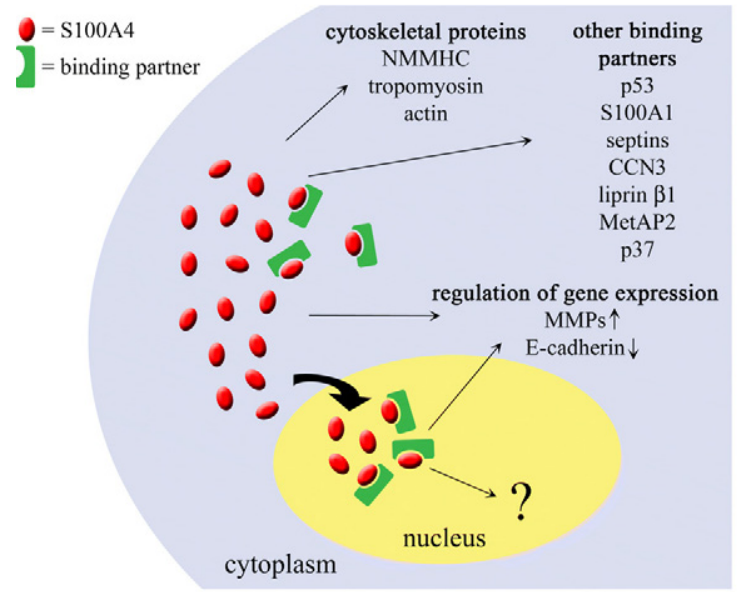

B

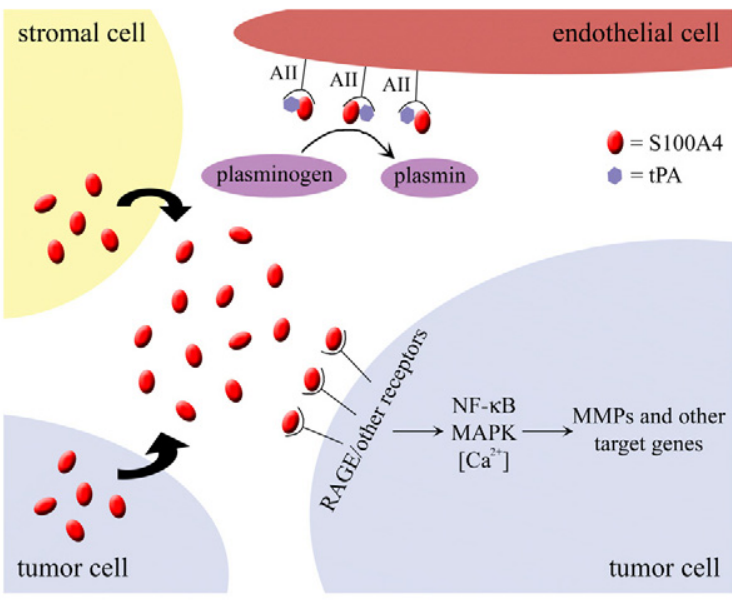

C

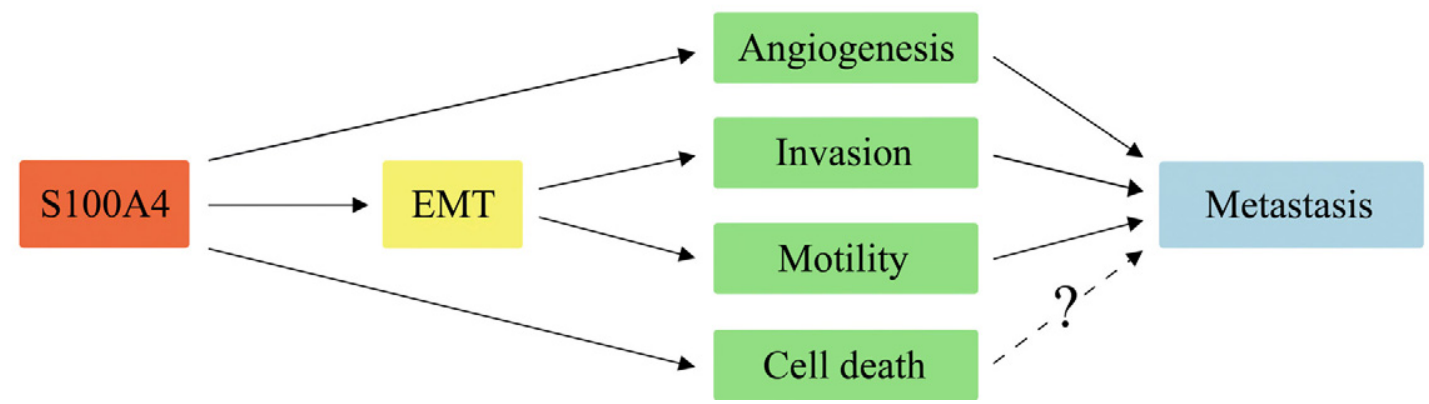

Figure 1. Mechanisms involved in S100A4-mediated metastatic progression. A: Molecular mechanisms associated with intracellular functions of S100A4. S100A4 is able to interact with cytoskeletal proteins, particularly nonmuscle myosin heavy chain (NMMHC) IIA, resulting in increased cell migration. In vitro studies have identified several other binding partners for S100A4 (upper right). However, the majority of these interactions have not been confirmed in vivo, and whether the interacting proteins are involved in S100A4-induced metastasis is thus mostly not known. Intracellular S100A4 expression is also associated with transcriptional regulation of certain genes, such as MMPs and E-cadherin, through so far unidentified mechanisms. Whether this could be attributed to cytoplasmic or nuclear S100A4 (or both) is also not known. Apart from the suggested functions mentioned above, the biological role of nuclear S100A4 still remains uncharacterized (question mark). B: Molecular mechanisms associated with extracellular S100A4. S100A4 is released from both tumor cells and stromal cells. Through interaction with annexin II (AII) and tissue plasminogen activator (tPA) on the surface of endothelial cells, S100A4 stimulates the conversion of plasminogen to plasmin, thus promoting angiogenesis. S100A4 also interacts with other cell surface receptors on both tumor cells and stromal cells (exemplified by a tumor cell in the figure), such as RAGE, to activate intracellular signal transduction cascades, including mitogen-activated protein kinases, increased intracellular $\left[\mathrm{Ca}^{2+}\right]$, and nuclear factor- $\kappa \mathrm{B}$ (see text for further details). S100A4-activated signaling pathways may, in turn, result in regulation of several target genes probably involved in stimulation of metastasis. The schematic illustrations provided in $\mathbf{A}$ and $\mathbf{B}$ are not comprehensive overviews of the molecular mechanisms of S100A4 but are illustrations of certain mechanisms associated with S100A4-induced stimulation of metastasis. C: Biological processes associated with S100A4. S100A4 stimulates cell motility, invasion, and angiogenesis and participates in the regulation of cell death Invasion and motility is probably promoted through induction of EMT. Cell motility, invasion, and angiogenesis all contribute to stimulation of metastasis, whereas the role of S100A4-mediated regulation of cell death in cancer metastasis is so far uncertain (dashed arrow).

ically normal and exhibited no increased frequency of neoplastic transformation in any organ. ${ }^{36,37}$ When these transgenic mice were crossed into a tumorigenic background, the offspring displayed a markedly increased frequency of lung metastasis, even though they developed primary tumors with incidence and tumor size comparable to their nontransgenic littermates. Furthermore, mice carrying null alleles for S100A4 demonstrated a significant attenuation of metastasis formation when crossed with PyV-mT mice that spontaneously develop multifocal mammary carcinomas and lung metastases. ${ }^{38}$ Using PyV-mT mice crossed with mice expressing green fluorescent protein (GFP) under control of the S100A4 promoter, the authors further showed that tumor cells leaving the primary tumor to invade neighboring structures turned GFP-positive (ie, activated the S100A4 promoter), and injection of disaggregated GFP-positive tumor cells from the primary tumors generated more metastases in the lungs and liver than injection of GFP-negative cells. In line with these observations, conditional apoptosis of cells in which the S100A4 promoter was active resulted in significant attenuation of lung metastases.

Altogether, the above-mentioned studies provide compelling evidence that S100A4 is directly involved in the formation of metastasis from several different tumor types. Common to most of these model systems is the fact that S100A4 does not affect the initiation and growth of the primary tumors, ${ }^{33,35-37}$ illustrating that S100A4 probably regulates steps in the metastatic cascade that do not impinge on tumor growth and proliferation.

\section{Prognostic Significance of S100A4}

Metastasis is the main cause of death in patients with cancer, and if the presence of S100A4 in tumor cells were 
as important in humans as in rodents, expression of S100A4 in primary tumors should robustly predict patient outcome. In line with this reasoning, numerous studies have investigated the potential use of S100A4 as a prognostic marker. In a panel of 349 patients with breast cancer, Rudland et al ${ }^{22}$ identified S100A4 protein expression as the most significant predictor of patient survival, even when compared with well-established markers of disease progression. Of patients with S100A4-negative tumors, $80 \%$ were alive after 19 years of follow-up, whereas only $11 \%$ of those with S100A4-positive tumors were still alive. These results were recently confirmed in two separate cohorts of patients, although the survival advantages in the S100A4-negative groups in these reports were less pronounced. ${ }^{13,39}$ Conflicting results have also been published, ${ }^{21}$ but these discrepancies could be explained by fewer patients, a shorter observation period, and differences in stage distribution and fixation methods. S100A4 is also overexpressed in colorectal cancer, ${ }^{8,23}$ and both mRNA and protein expression are correlated with patient outcome. ${ }^{8,40}$ Furthermore, an association between S100A4 protein expression and patient survival has been shown in several other tumor types, such as ovarian carcinoma, pancreatic cancer, malignant melanoma, bladder cancer, non-small cell lung cancer, gallbladder cancer, esophageal squamous cell carcinoma, and gastric cancer. ${ }^{2,14}$

Importantly, all investigations examining the prognostic significance of S100A4 expression have so far been retrospectively conducted studies. Preliminary analyses of the follow-up data from our panel of prospectively collected tumor samples from patients with colorectal cancer indicate that nuclear expression of S100A4 is an independent predictor of patient survival (K. Boye and $\mathrm{K}$. Flatmark, manuscript in preparation), but to resolve the issue of whether immunohistochemical staining for S100A4 could be an adjunct to clinicopathological staging in routine clinical practice, further prospectively designed investigations on large patient cohorts are certainly warranted.

\section{Mechanisms of S100A4-Induced Metastatic Progression}

As illustrated in the previous sections, S100A4 is a well established marker and mediator of metastatic disease, but the exact mechanisms responsible for the metastasispromoting effects are less well defined. In the following, some of the functions of S100A4 are summarized, with an emphasis on the mechanisms connecting S100A4 and cancer metastasis (Figure 1, A-C).

\section{Motility and Invasion}

Transfection studies using several different cell systems have convincingly shown that S100A4 is involved in stimulation of cell motility. $8,12,34,41$ In support of this finding, S100A4 expression in normal cell types is almost exclusively restricted to cells with a motile phenotype, such as leukocytes and fibroblasts. The interaction with nonmuscle myosin IIA is essential for S100A4-induced migration, and several groups have shown that addition of S100A4 inhibits assembly of myosin filaments and even promotes disassembly of preformed filaments. ${ }^{26} \mathrm{Re}$ cently, S100A4 was also shown to colocalize with myosin IIA at the leading edge of migrating cells ${ }^{42}$ and to promote directional motility through a direct interaction with myosin IIA. ${ }^{43}$ Furthermore, mutations in the C-terminal region of S100A4 resulted in decreased binding to myosin IIA, and cells expressing these mutants were less motile and invasive in vitro and less metastatic in vivo. ${ }^{44}$ Taken together, these studies suggest that S100A4 enhances the turnover of myosin IIA filaments at the leading edge of migrating cells, resulting in increased motility, which could contribute to an increased metastatic capacity of cancer cells.

The ability to migrate is a prerequisite for a cancer cell to escape the primary tumor and enter the circulation. However, to travel through the surrounding stroma, these locally invading cells must be able to proteolytically degrade extracellular matrix components. Matrix metalloproteinases (MMPs) play an essential role in this process, and several experimental strategies have established an intimate connection between S100A4 and certain members of the MMP family. Down-regulation of S100A4 expression in osteosarcoma cells led to reduced expression of MMP-2 and MT1-MMP, with a subsequent reduction in MMP-2 activity and a reduced ability to migrate through Matrigel-coated filters. ${ }^{41}$ The invasive capability of human prostate cancer cells is also stimulated by S100A4, at least partly through transcriptional activation of MMP-9. ${ }^{45}$ Moreover, induction of S100A4 expression by gain-of-function $\beta$-catenin mutations conferred invasive capabilities to human colorectal cancer cell lines in vitro and in vivo, ${ }^{8}$ but the impact of S100A4 expression on MMP activation was not investigated in this study.

The mechanisms by which S100A4 participates in the regulation of MMPs are mostly unknown, but recent studies suggest that extracellular activities are involved. ${ }^{12,18,28,46}$ Addition of recombinant S100A4 to the culture medium of tumor cells, endothelial cells, chondrocytes, and synovial fibroblasts produced a significant increase in MMP-13 levels and also altered the expression levels of certain other MMPs. In the previously mentioned investigations on tumor cells, whether intracellular levels and/or autocrine actions of extracellular S100A4 contributed to the observed MMP regulation was not examined. However, we were unable to detect any induction of MMP-2 or other MMPs in osteosarcoma cell lines upon treatment with extracellular $\mathrm{S} 100 \mathrm{~A} 4,{ }^{47}$ suggesting that intracellular functions of S100A4 are involved, at least in certain cell systems.

Still, even though an intimate connection between S100A4 and various members of the MMP family seems to exist, no conclusive evidence has so far been provided to implicate MMPs in any of the experimental models of S100A4-induced metastasis. 


\section{Angiogenesis}

Based on observations in transgenic mice, S100A4 has been identified as a potent stimulator of angiogenesis. Aging S100A4 transgenic mice developed hemangiomas in the liver with a significantly increased incidence compared with nontransgenic animals, and micropellets containing S100A4 induced neovascularization when implanted in the corneas of mice. ${ }^{15}$ Importantly, S100A4 expression is associated with angiogenesis in neoplastic lesions also, as mammary tumors from S100A4 transgenic mice displayed higher vessel density compared with nontransgenic animals, ${ }^{15}$ and vessel density and S100A4 expression were positively correlated in primary tumors from patients with breast cancer. ${ }^{13}$ The mechanisms that participate in this process are only starting to become unraveled. Augmented MMP-13 expression in endothelial cells was associated with S100A4-mediated stimulation of capillary-like growth in three-dimensional Matrigel cultures in vitro, ${ }^{46}$ and through interaction with annexin II, extracellular S100A4 accelerated tissue plasminogen activator-mediated conversion of plasminogen to plasmin, resulting in capillary-like tube formation of human cerebromicrovascular endothelial cells. ${ }^{10}$ Interestingly, S100A4-stimulated plasmin activation may also contribute to the observed activation of MMP-2 and MMP13. However, it seems unlikely that annexin II binding or plasminogen conversion would result in increased transcription of several MMPs.

\section{Role of the Tumor Stroma}

Even though extracellular S100A4 is increasingly recognized as a key player in tumor progression and metastasis, two key questions remain largely unanswered: which cell types are the key producers of extracellular S100A4 and which receptor(s) and signal transduction mechanisms mediate the prometastatic actions of this multifaceted protein? Both tumor cells and nonmalignant cells present in the tumor stroma secrete S100A4 (see Subcellular Localization and Tissue Distribution), and tumor cells have also been shown to stimulate the release of S100A4 from stromal cells. ${ }^{18}$ Seemingly, S100A4 promotes metastatic progression by influencing both stromal cells and tumor cells, and the essential prerequisite might be the mere presence of S100A4 in the tumor microenvironment rather than its specific release from certain cell types. S100A4 exists in several functional states, of which dimeric and oligomeric forms are most common, and the latter seem to be associated with extracellular activity. ${ }^{15,29}$ Whether different cells produce dissimilar functional forms of S100A4 is currently not known; however, both tumor cell-derived and stroma-derived S100A4 possess metastasis-promoting properties. The essential role of the S100A4-expressing stroma was recently demonstrated using two separate experimental models: i) S100A4-negative tumor cells coinjected with recombinant S100A4 protein showed an increased metastatic capacity compared with tumor cells co-injected with a recombinant fragment of myosin heavy chain or tumor cells alone ${ }^{18}$; and ii) S100A4 knockout mice injected with highly metastatic breast cancer cells did not develop any metastases, whereas all wild-type control animals showed signs of metastatic disease.48 Co-injection of S100A4-positive fibroblasts partly rescued the attenuated metastasis formation, substantiating the metastasis-promoting effects of S100A4-positive cells in the tumor microenvironment.

\section{Cell Surface Receptors and Intracellular Signal Transduction}

Most likely, the biological effects of extracellular S100A4 are mediated through a cell surface receptor, ${ }^{14}$ and as for other S100 proteins, RAGE has been suggested as a putative receptor. Indeed, RAGE-mediated translocation of intracellular S100A4 in response to the extracellular protein has been demonstrated, ${ }^{11}$ and S100A4-mediated signaling in human chondrocytes is RAGE-dependent. ${ }^{28}$ However, RAGE-negative cell lines are responsive to both S100A4-induced stimulation of cell motility ${ }^{49}$ and capillary-like growth, ${ }^{46}$ and S100A4-mediated neurite outgrowth was recently shown to be RAGE-independent. ${ }^{27}$ As for S100A8 and S100A9, binding of S100A4 to heparan sulfate moieties on the cell surface has been reported, ${ }^{27}$ possibly acting as a scaffold to bring $S 100$ proteins in close vicinity to their specific, signal-transducing receptors. Interestingly, other S100 proteins also display both RAGE-dependent and -independent actions, which might imply that different functional states of the S100 proteins (eg, dimeric and oligomeric forms) could be recognized by different receptors. As mentioned above, S100A4 also interacts with annexin II on the surface of endothelial cells. ${ }^{10}$ In this setting, annexin II acts as a coreceptor governing the assembly of S100A4, plasminogen and its activators, but it is not known to be responsible for the propagation of intracellular signal transduction cascades. Altogether, these data demonstrate that RAGE and annexin II mediate some of the extracellular functions of S100A4; however, S100A4 interacts with and exerts several of its effects through an as yet unidentified cell surface receptor.

The signal transduction pathways involved after receptor ligation are also incompletely characterized, perhaps with the exception of signaling events leading to neurite outgrowth. In this case, S100A4 activates a cascade involving heterotrimeric $\mathrm{G}$ proteins, phospholipase $\mathrm{C}$, protein kinase $\mathrm{C}$, elevation of intracellular $\left[\mathrm{Ca}^{2+}\right]$ and extracellular signalregulated kinases 1 and 2. ${ }^{27,29}$ In other cell systems, including cells present in the tumor microenvironment, extracellular S100A4 has been shown to activate the transcription factor nuclear factor- $\kappa \mathrm{B},{ }^{11,17,28,46}$ indicating that nuclear factor- $\kappa \mathrm{B}$ activation and subsequent regulation of target genes might be involved in S100A4mediated metastatic progression. ${ }^{47}$ Furthermore, preliminary data from our laboratory indicate that extracellular S100A4 is internalized by several cancer cell lines (K. Andersen and G. M. Mælandsmo, unpublished observations), and similar results have been obtained using cardiac myocytes. ${ }^{5}$ These observations provide evidence of 
yet another means by which S100A4 may exert its effects on target cells.

\section{Regulation of Cell Death}

Finally, both extracellular and intracellular S100A4 participates in the regulation of cell death. Prosurvival functions have been described both in malignant ${ }^{50}$ and nonmalignant cell systems, ${ }^{5}$ whereas osteosarcoma cells were sensitized to apoptosis on treatment with extracellular S100A4. ${ }^{17}$ Furthermore, we and others have observed increased apoptosis in cells transfected with S100A4, ${ }^{51}$ and on this basis Grigorian et $\mathrm{al}^{51}$ performed a search for binding partners mediating this increased cell death. A series of in vitro experiments revealed that S100A4 binds the C-terminal end of p53 and modulates transcription of p53-responsive genes, such as mdm2, Bax, p21 and thrombospondin-1. Whether S100A4 interacts with p53 in the cytoplasm and/or in the nucleus is still not known, and, similarly, the function of nuclear S100A4 in both normal and malignant cells remains completely enigmatic. Recently, it was demonstrated using S100A4 knockout mice that S100A4 might cooperate with p53 to induce apoptosis also in vivo. ${ }^{52} \mathrm{~S} 100 \mathrm{~A} 4^{-1-}$ mice are viable and phenotypically normal in the postnatal period, but $\sim 10 \%$ of the animals develop tumors at age 10 to 14 months. The authors speculated that this could be explained by loss of p53 tumor suppressor activity due to impaired interaction with S100A4. Thus, S100A4 may act both as a tumor suppressor and a metastasis promoter, similar to the epithelial-mesenchymal transition (EMT)-inducing cytokine transforming growth factor- $\beta$, which plays an important role in early tumor suppression, but stimulates tumor progression once the tumor is established.

\section{S100A4 and Epithelial-Mesenchymal Transition}

Metastasizing cells must alter their phenotype to facilitate movement and local invasion, a process similar to the EMT observed in embryonic development. On these grounds, it has been hypothesized that carcinoma cells undergo EMT to facilitate metastasis, and convincing experimental evidence, at least from in vitro studies, has recently been provided. ${ }^{53}$ However, observations of EMT in vivo are scarce, and the role of EMT in cancer metastasis is thus highly debated. ${ }^{54}$ In recent years, it has been hypothesized that some of the prometastatic actions of S100A4 could be explained by its role in epithelial-mesenchymal transition.

The evidence connecting S100A4 and EMT comes from studies on renal fibrosis, in which S100A4 was identified as a fibroblast-specific protein and renamed FSP1. ${ }^{19}$ Transfection of S100A4 into murine proximal tubular epithelial cells induced EMT, ${ }^{19}$ and when S100A4 expression was blocked by antisense molecules, the renal epithelial cells were not able to accomplish cytokineinduced EMT. ${ }^{5}$
In the study by Xue et $\mathrm{al}^{38}$ mentioned previously, it was elegantly and convincingly shown that S100A4 is functionally involved in the development of lung metastasis in PyV-mT mice. Based on the experimental data provided, it seems plausible that S100A4 facilitates EMT to promote metastatic dissemination in this animal model; however, this hypothesis is not substantiated by the concomitant expression of molecular markers of EMT in the metastasizing tumor cells, such as reduced levels of epithelial markers or gain of mesenchymal markers. To our knowledge, no conclusive proof exists to causally implicate S100A4 in EMT in models of cancer progression. Nevertheless, the circumstantial evidence and the indications presented in numerous studies over more than a decade are overwhelming.

First, S100A4 and E-cadherin are inversely regulated in several cell systems, and overexpression of S100A4 generally inhibits E-cadherin expression and vice versa. ${ }^{55} \mathrm{Sec}$ ond, accumulation of nuclear $\beta$-catenin has been observed at the invasive fronts and in tumor cells migrating into the surrounding stroma, ${ }^{56}$ implying that $\beta$-catenindriven S100A4-dependent induction of tumor cell motility and invasiveness may reflect initiation of EMT. ${ }^{8}$ Third, the key transcriptional EMT-regulator Snail was reported to induce S100A4 expression in tumors undergoing EMT. ${ }^{57}$ Fourth, as summarized above, S100A4 directly promotes the expression of several MMPs, a hallmark of epithelial cells undergoing a mesenchymal transition. Fifth, S100A4 activates nuclear factor $-\kappa \mathrm{B}$, which is involved in the EMT transcriptional program by up-regulating essential transcription factors, such as Twist and Snail, as well as mesenchymal markers, including vimentin and proteases. Last and most importantly, given the assumption that EMT is a common mechanism for promotion of cell migration and invasiveness, the convincing evidence connecting S100A4 to a motile and invasive phenotype in numerous cell systems highly suggests S100A4 as a key player in EMT induction.

\section{Conclusions}

The evidence implicating S100A4 in cancer metastasis is compelling, and S100A4 plays an important role in the metastatic journey of tumor cells by influencing several steps in the metastatic cascade, including migration, invasiveness, and angiogenesis. The molecular mechanisms by which S100A4 exerts these functions remain unclear, and considerable functional diversity is created by the fact that extracellular, intracellular, tumor-derived, and stroma-derived S100A4 all contribute to metastatic dissemination. Intriguingly, S100A4-mediated induction of EMT may explain several of the prometastatic actions of S100A4, and S100A4 seems to promote disease progression through similar biological mechanisms also in other, nonmalignant conditions. ${ }^{5}$

Obviously, a more comprehensive understanding of the molecular mechanisms by which S100A4 promotes metastasis is needed, and this must be a central focus of future research efforts. Another priority area in S100A4 research should be the discovery of novel treatment strat- 
egies targeting S100A4, as well as the further development of suggested inhibitors, which hopefully will bring anti-S100A4 compounds into clinical trials in the not-sodistant future.

\section{Acknowledgment}

We thank Professor Øystein Fodstad for critical reading of the manuscript. We apologize to all authors whose work could not be cited because of space limitations.

\section{References}

1. Garrett SC, Varney KM, Weber DJ, Bresnick AR: S100A4, a mediator of metastasis. J Biol Chem 2006, 281:677-680

2. Mazzucchelli L: Protein S100A4: too long overlooked by pathologists? Am J Pathol 2002, 160:7-13

3. Marenholz I, Lovering RC, Heizmann CW: An update of the $\mathrm{S} 100$ nomenclature. Biochim Biophys Acta 2006, 1763:1282-1283

4. Donato R: Intracellular and extracellular roles of S100 proteins. Microsc Res Tech 2003, 60:540-551

5. Schneider M, Hansen JL, Sheikh SP: S100A4: a common mediator of epithelial-mesenchymal transition, fibrosis and regeneration in diseases? J Mol Med 2008, 86:507-522

6. Malashkevich VN, Varney KM, Garrett SC, Wilder PT, Knight D, Charpentier TH, Ramagopal UA, Almo SC, Weber DJ, Bresnick AR: Structure of $\mathrm{Ca}^{2+}$-bound S100A4 and its interaction with peptides derived from nonmuscle myosin-IIA. Biochemistry 2008, 47:5111-5126

7. Cohn MA, Hjelmso I, Wu LC, Guldberg P, Lukanidin EM, Tulchinsky EM: Characterization of Sp1, AP-1, CBF and KRC binding sites and minisatellite DNA as functional elements of the metastasis-associated mts $1 /$ S100A4 gene intronic enhancer. Nucleic Acids Res 2001, 29:3335-3346

8. Stein $U$, Arlt F, Walther W, Smith J, Waldman T, Harris ED, Mertins SD, Heizmann CW, Allard D, Birchmeier W, Schlag PM, Shoemaker RH: The metastasis-associated gene S100A4 is a novel target of $\beta$-catenin/T-cell factor signaling in colon cancer. Gastroenterology 2006, 131:1486-1500

9. Flatmark K, Pedersen KB, Nesland JM, Rasmussen H, Aamodt G, Mikalsen SO, Bjornland K, Fodstad O, Maelandsmo GM: Nuclear localization of the metastasis-related protein S100A4 correlates with tumour stage in colorectal cancer. J Pathol 2003, 200:589-595

10. Semov A, Moreno MJ, Onichtchenko A, Abulrob A, Ball M, Ekiel I, Pietrzynski G, Stanimirovic D, Alakhov V: Metastasis-associated protein S100A4 induces angiogenesis through interaction with Annexin II and accelerated plasmin formation. J Biol Chem 2005, 280:20833-20841

11. Hsieh HL, Schafer BW, Weigle B, Heizmann CW: S100 protein translocation in response to extracellular $\mathrm{S} 100$ is mediated by receptor for advanced glycation endproducts in human endothelial cells. Biochem Biophys Res Commun 2004, 316:949-959

12. Kikuchi N, Horiuchi A, Osada R, Imai T, Wang C, Chen X, Konishi I: Nuclear expression of S100A4 is associated with aggressive behavior of epithelial ovarian carcinoma: an important autocrine/paracrine factor in tumor progression. Cancer Sci 2006, 97:1061-1069

13. de Silva Rudland S, Martin L, Roshanlall C, Winstanley J, Leinster S, Platt-Higgins A, Carroll J, West C, Barraclough R, Rudland P: Association of S100A4 and osteopontin with specific prognostic factors and survival of patients with minimally invasive breast cancer. Clin Cancer Res 2006, 12:1192-1200

14. Helfman DM, Kim EJ, Lukanidin E, Grigorian M: The metastasis associated protein S100A4: role in tumour progression and metastasis. Br J Cancer 2005, 92:1955-1958

15. Ambartsumian N, Klingelhofer J, Grigorian M, Christensen C, Kriajevska M, Tulchinsky E, Georgiev G, Berezin V, Bock E, Rygaard J, Cao R, Cao Y, Lukanidin E: The metastasis-associated Mts1(S100A4) protein could act as an angiogenic factor. Oncogene 2001, 20:4685-4695

16. Cabezón T, Celis JE, Skibshoj I, Klingelhofer J, Grigorian M, Gromov P, Rank F, Myklebust JH, Maelandsmo GM, Lukanidin E, Ambartsumian $\mathrm{N}$ : Expression of S100A4 by a variety of cell types present in the tumor microenvironment of human breast cancer. Int $\mathrm{J}$ Cancer 2007, 121:1433-1444

17. Pedersen KB, Andersen K, Fodstad O, Maelandsmo GM: Sensitization of interferon-gamma induced apoptosis in human osteosarcoma cells by extracellular S100A4. BMC Cancer 2004, 4:52

18. Schmidt-Hansen B, Klingelhofer J, Grum-Schwensen B, Christensen A, Andresen S, Kruse C, Hansen T, Ambartsumian N, Lukanidin E, Grigorian M: Functional significance of metastasis-inducing S100A4(Mts1) in tumor-stroma interplay. J Biol Chem 2004, 279:24498-24504

19. Strutz F, Okada H, Lo CW, Danoff T, Carone RL, Tomaszewski JE, Neilson EG: Identification and characterization of a fibroblast marker: FSP1. J Cell Biol 1995, 130:393-405

20. Takenaga K, Nakamura Y, Sakiyama S: Cellular localization of pEL98 protein, an $\mathbf{5 1 0 0 - r e l a t e d ~ c a l c i u m ~ b i n d i n g ~ p r o t e i n , ~ i n ~ f i b r o b l a s t s ~ a n d ~ i t s ~}$ tissue distribution analyzed by monoclonal antibodies. Cell Struct Funct 1994, 19:133-141

21. Pedersen KB, Nesland JM, Fodstad O, Maelandsmo GM: Expression of S100A4. E-cadherin, $\alpha$ - and $\beta$-catenin in breast cancer biopsies, Br J Cancer 2002, 87:1281-1286

22. Rudland PS, Platt-Higgins A, Renshaw C, West CR, Winstanley JH, Robertson L, Barraclough R: Prognostic significance of the metastasis-inducing protein S100A4 (p9Ka) in human breast cancer. Cancer Res 2000, 60:1595-1603

23. Takenaga K, Nakanishi H, Wada K, Suzuki M, Matsuzaki O, Matsuura A, Endo H: Increased expression of S100A4, a metastasis-associated gene, in human colorectal adenocarcinomas. Clin Cancer Res 1997, 3:2309-2316

24. Grigorian M, Tulchinsky E, Burrone O, Tarabykina S, Georgiev G, Lukanidin E: Modulation of mts1 expression in mouse and human normal and tumor cells. Electrophoresis 1994, 15:463-468

25. Tarabykina S, Griffiths TR, Tulchinsky E, Mellon JK, Bronstein IB, Kriajevska M: Metastasis-associated protein S100A4: spotlight on its role in cell migration. Curr Cancer Drug Targets 2007, 7:217-228

26. Santamaria-Kisiel L, Rintala-Dempsey AC, Shaw GS: Calcium-dependent and -independent interactions of the S100 protein family. Biochem J 2006, 396:201-214

27. Kiryushko D, Novitskaya V, Soroka V, Klingelhofer J, Lukanidin E, Berezin V, Bock E: Molecular mechanisms of $\mathrm{Ca}^{2+}$ signaling in neurons induced by the S100A4 protein. Mol Cell Biol 2006, 26:36253638

28. Yammani RR, Carlson CS, Bresnick AR, Loeser RF: Increase in production of matrix metalloproteinase 13 by human articular chondrocytes due to stimulation with S100A4: role of the receptor for advanced glycation end products. Arthritis Rheum 2006, 54:2901-2911

29. Novitskaya V, Grigorian M, Kriajevska M, Tarabykina S, Bronstein I, Berezin V, Bock E, Lukanidin E: Oligomeric forms of the metastasisrelated Mts1 (S100A4) protein stimulate neuronal differentiation in cultures of rat hippocampal neurons. J Biol Chem 2000, 275:41278-41286

30. Ebralidze A, Tulchinsky E, Grigorian M, Afanasyeva A, Senin V, Revazova E, Lukanidin E: Isolation and characterization of a gene specifically expressed in different metastatic cells and whose deduced gene product has a high degree of homology to a $\mathrm{Ca}^{2+}$. binding protein family. Genes Dev 1989, 3:1086-1093

31. Davies BR, Davies MP, Gibbs FE, Barraclough R, Rudland PS: Induction of the metastatic phenotype by transfection of a benign rat mammary epithelial cell line with the gene for p9Ka, a rat calciumbinding protein, but not with the oncogene EJ-ras-1. Oncogene 1993, 8:999-1008

32. Grigorian M, Ambartsumian N, Lykkesfeldt AE, Bastholm L, Elling F, Georgiev G, Lukanidin E: Effect of mts1 (S100A4) expression on the progression of human breast cancer cells. Int J Cancer 1996, 67:831-841

33. Maelandsmo GM, Hovig E, Skrede M, Engebraaten O, Florenes VA, Myklebost O, Grigorian M, Lukanidin E, Scanlon KJ, Fodstad O: Reversal of the in vivo metastatic phenotype of human tumor cells by an anti-CAPL (mts1) ribozyme. Cancer Res 1996, 56:5490-5498

34. Takenaga K, Nakamura Y, Sakiyama S: Expression of antisense RNA to S100A4 gene encoding an S100-related calcium-binding protein suppresses metastatic potential of high-metastatic Lewis lung carcinoma cells. Oncogene 1997, 14:331-337

35. Levett D, Flecknell PA, Rudland PS, Barraclough R, Neal DE, Mellon JK, Davies BR: Transfection of S100A4 produces metastatic variants of an orthotopic model of bladder cancer. Am J Pathol 2002, 160:693-700

36. Ambartsumian NS, Grigorian MS, Larsen IF, Karlstrom O, Sidenius N, 
Rygaard J, Georgiev G, Lukanidin E: Metastasis of mammary carcinomas in GRS/A hybrid mice transgenic for the mts1 gene. Oncogene 1996, 13:1621-1630

37. Davies MP, Rudland PS, Robertson L, Parry EW, Jolicoeur P, Barraclough R: Expression of the calcium-binding protein S100A4 (p9Ka) in MMTV-neu transgenic mice induces metastasis of mammary tumours. Oncogene 1996, 13:1631-1637

38. Xue C, Plieth D, Venkov C, Xu C, Neilson EG: The gatekeeper effect of epithelial-mesenchymal transition regulates the frequency of breast cancer metastasis. Cancer Res 2003, 63:3386-3394

39. Lee WY, Su WC, Lin PW, Guo HR, Chang TW, Chen HH: Expression of S100A4 and Met: potential predictors for metastasis and survival in early-stage breast cancer. Oncology 2004, 66:429-438

40. Gongoll S, Peters G, Mengel M, Piso P, Klempnauer J, Kreipe H, von Wasielewski R: Prognostic significance of calcium-binding protein S100A4 in colorectal cancer. Gastroenterology 2002, 123:1478-1484

41. Biørnland K, Winberg JO, Odegaard OT, Hovig E, Loennechen T, Aasen AO, Fodstad O, Maelandsmo GM: S100A4 involvement in metastasis: deregulation of matrix metalloproteinases and tissue inhibitors of matrix metalloproteinases in osteosarcoma cells transfected with an anti-S100A4 ribozyme. Cancer Res 1999, 59:47024708

42. Kim EJ, Helfman DM: Characterization of the metastasis-associated protein. S100A4 roles of calcium binding and dimerization in cellular localization and interaction with myosin, J Biol Chem 2003, 278:30063-30073

43. Li ZH, Bresnick AR: The S100A4 metastasis factor regulates cellular motility via a direct interaction with myosin-IIA. Cancer Res 2006, 66:5173-5180

44. Ismail T, Fernig DG, Rudland PS, Terry CJ, Wang G, Barraclough R: The basic C-terminal amino acids of calcium-binding protein S100A4 promote metastasis. Carcinogenesis 2008, 29:2259-2266

45. Saleem M, Kweon MH, Johnson JJ, Adhami VM, Elcheva I, Khan N, Bin Hafeez B, Bhat KM, Sarfaraz S, Reagan-Shaw S, Spiegelman VS, Setaluri V, Mukhtar H: S100A4 accelerates tumorigenesis and invasion of human prostate cancer through the transcriptional regulation of matrix metalloproteinase 9. Proc Natl Acad Sci USA 2006, 103:14825-14830

46. Schmidt-Hansen B, Ornas D, Grigorian M, Klingelhofer J, Tulchinsky E, Lukanidin E, Ambartsumian N: Extracellular S100A4(mts1) stimulates invasive growth of mouse endothelial cells and modulates MMP-13 matrix metalloproteinase activity. Oncogene 2004, 23: $5487-5495$
47. Boye K, Grotterod I, Aasheim HC, Hovig E, Maelandsmo GM: Activation of NF- $\kappa$ B by extracellular S100A4: analysis of signal transduction mechanisms and identification of target genes. Int $\mathrm{J}$ Cancer 2008, 123:1301-1310

48. Grum-Schwensen B, Klingelhofer J, Berg CH, El-Naaman C, Grigorian M, Lukanidin E, Ambartsumian N: Suppression of tumor development and metastasis formation in mice lacking the $\mathrm{S} 100 \mathrm{~A} 4$ (mts1) gene. Cancer Res 2005, 65:3772-3780

49. Belot N, Pochet R, Heizmann CW, Kiss R, Decaestecker C: ExtracelIular S100A4 stimulates the migration rate of astrocytic tumor cells by modifying the organization of their actin cytoskeleton. Biochim Biophys Acta 2002, 1600:74-83

50. Mahon PC, Baril P, Bhakta V, Chelala C, Caulee K, Harada T, Lemoine NR: S100A4 contributes to the suppression of BNIP3 expression, chemoresistance, and inhibition of apoptosis in pancreatic cancer. Cancer Res 2007, 67:6786-6795

51. Grigorian M, Andresen S, Tulchinsky E, Kriajevska M, Carlberg C, Kruse C, Cohn M, Ambartsumian N, Christensen A, Selivanova G, Lukanidin E: Tumor suppressor p53 protein is a new target for the metastasis-associated Mts1/S100A4 protein: functional consequences of their interaction. J Biol Chem 2001, 276:22699-22708

52. EL Naaman C, Grum-Schwensen B, Mansouri A, Grigorian M, SantoniRugiu E, Hansen T, Kriajevska M, Schafer BW, Heizmann CW, Lukanidin E, Ambartsumian N: Cancer predisposition in mice deficient for the metastasis-associated Mts1(S100A4) gene. Oncogene 2004, 23:3670-3680

53. Hugo H, Ackland ML, Blick T, Lawrence MG, Clements JA, Williams ED, Thompson EW: Epithelial-mesenchymal and mesenchymal-epithelial transitions in carcinoma progression. J Cell Physiol 2007, 213:374-383

54. Tarin D, Thompson EW, Newgreen DF: The fallacy of epithelial mesenchymal transition in neoplasia. Cancer Res 2005, 65:5996-6000; discussion 6000-5991

55. Keirsebilck A, Bonne S, Bruyneel E, Vermassen P, Lukanidin E, Mareel M, van Roy F: E-cadherin and metastasin (mts-1/S100A4) expression levels are inversely regulated in two tumor cell families. Cancer Res 1998, 58:4587-4591

56. Brabletz T, Jung A, Spaderna S, Hlubek F, Kirchner T: Opinion: migrating cancer stem cells-an integrated concept of malignant tumour progression. Nat Rev Cancer 2005, 5:744-749

57. Moody SE, Perez D, Pan TC, Sarkisian CJ, Portocarrero CP, Sterner CJ, Notorfrancesco KL, Cardiff RD, Chodosh LA: The transcriptional repressor Snail promotes mammary tumor recurrence. Cancer Cell 2005, 8:197-209 